\title{
Briefs
}

\section{Limiting Efficiencies of GaAs Solar Cells}

GERARDO L. ARAÚJO AND ANTONIO MARTÍ

Abstract-The limiting efficiencies of GaAs solar cells when used under concentrated sunlight are calculated. An assessment of the benefits to be expected from applying techniques which restrict the angle of acceptance of the cell is made. It is concluded that by restricting the acceptance angle the emission of the luminescent photons and therefore the associated current loss are reduced. As a consequence of this effect, a limiting efficiency close to $39 \%$ results for concentration ratios of about 1000 suns AM1.5 Direct. For lower concentration ratios, the limiting efficiency decreases if Auger recombination is also taken into account.

\section{INTRODUCTION}

The limiting efficiency of semiconductor solar cells has been calculated in the past from first principles [1]-[3], considering that losses are due to radiative recombination processes only.

It has been demonstrated, however, that Si solar cells are limited by Auger recombination, rather than by radiative recombination [4]-[7]. To reduce Auger recombination, very thin Si cells (20-50 $\mu \mathrm{m})$ are needed, together with the use of light-trapping techniques [5]. And even thinner devices could be used, with still further improvements predicted, if techniques which restrict the angle of acceptance of the incoming light [6]-[8] were employed.

It was the purpose of this work to make a quantitative assessment of the benefits to be expected from applying to GaAs cells similar techniques of restriction of the acceptance angle to that strictly necessary to collect the incoming light. It was found that this directbandgap semiconductor is limited by luminescent emission and therefore by restricting the acceptance angle the emission of luminescence photons and its associated current loss is decreased and, consequently, a gain in efficiency can be obtained, without the need of sophisticated trapping techniques.

\section{Theory AND CASES OF STUdy}

Our approach analyzes an idealized device which basically consists of an absorbing semiconductor layer characterized by its energy gap $E_{G}$, refractive index $n$, absorption coefficient $\alpha$, and thickness $W$. The quasi-Fermi levels are splitted by $q V$ uniformly, due to illumination and load conditions, consequently giving rise to current losses due to radiative and nonradiative recombination processes. The general expressions for the calculation of the solar cell characteristics are summarized in Table I. These expressions were obtained as a straightforward generalization of expressions in previous studies [3], [4], [6].

$S_{1}(E)$ is the incident solar photon radiance for a given one-sun spectrum, $X$ is the number of suns, $a(E, \varphi)$ is the absorbance of the semiconductor (taken as zero outside the solid angle of acceptance $2 \theta)$, and $b(E, T)$ is the flux of black-body photons, per unit energy and solid angle, corresponding to the air considered as the

Manuscript received July 29, 1988; revised October 25, 1989. This work was supported by the Spanish Comision Interministerial de Ciencia y Tecnologia (CICYT) under Project MC1/85. The review of this brief was arranged by Associate Editor S. J. Fonash.

The authors are with the Instituto de Energía Solar-ETSI. Telecomunicación, Universidad Politécnica de Madrid, 28040 Madrid, Spain.

IEEE Log Number 9034391 .
TABLE I

Summary of the Expressions Used in the Calculation of the Limiting EFFICIENCIES

\begin{tabular}{|c|c|}
\hline$J=X J_{L 1}-J_{1 e}-J_{a g}-J_{s r h}-J_{s p}$ & (1) \\
\hline $\begin{array}{l}\text { Photogenerated current at one sun, } J \text { : } \\
\qquad J_{L_{1}}=q \int_{E c}^{\infty} S_{1}(E) a(E) d E\end{array}$ & (2) \\
\hline $\begin{array}{l}\text { Luminescent current losses (for each surface) } \\
J_{1 e}=q \int_{0}^{\theta} \int_{E G}^{\infty} b(E, T) a(E, \varphi) \cos \varphi d \Omega d E\end{array}$ &,$J_{1 e}$ \\
\hline $\begin{array}{l}\text { Auger recombination losses, } J_{a q}: \\
\qquad J_{a q}=q W\left(C_{n} n+C_{p} p\right) n_{i}^{2} \exp (q V / k T)\end{array}$ & (4) \\
\hline $\begin{array}{l}\text { Via trap-level losses, } J_{s r h}: \\
J_{s i h}=q W \frac{n_{1}^{2}}{\tau\left(p+n+2 n_{i}\right)} \exp (q V / k T)\end{array}$ & (5) \\
\hline $\begin{array}{l}J_{s p}=q S \frac{n_{1}^{2}}{\left(p+n+2 n_{i}\right)} \exp (q V / k T)\end{array}$ & (6) \\
\hline
\end{tabular}

optical medium surrounding the cell. $C_{n}$ and $C_{p}$ are the Auger coefficients and $\tau$ and $S$ are the SRH lifetime and surface recombination velocity, respectively, assumed to be the same for both carriers. Besides the equations in Table I, the general relationships $p n=$ $n_{i}^{2} \exp (q V / k T)$ and the charge neutrality equation were used where necessary.

Two different structures have been analyzed: For that referred to as "D1-structure" the back surface of the device is a perfect reflector and therefore the radiation occurs through the front surface to the air. Such a situation can be approached, in practice, by a silicon solar cell. But the very thin active regions of GaAs solar cells must normally be epitaxially grown on a semiconductor substrate. Therefore, unless very effective post-processing thinning techniques are used, the substrate constitutes a photovoltaically passive optical medium at the back of the active device, having the same index of refraction and therefore the device will have luminescent losses because of radiation to the air and to the substrate. This case will be referred to as "D2-structure."

\section{Step Function Absorbance and Radiative} RECOMBINATION ONLY

It is illustrative to consider first the case for which the luminescent current losses are much higher than any other recombination losses. This case is important from a basic point of view and because it would happen to hold for GaAs solar cells, if via intermediate levels and surface recombination could be neglected.

In our calculations, the restriction of the acceptance angle is modeled by considering that the absorbance has a value of unity for the radiation reaching the cell within the acceptance angle, $2 \theta$, and zero outside that angle. This means that our devices have a perfect, angularly selective antireflective coating on its front surface.

The photo-generated current for our ideal devices $(a=1)$ exposed to one-sun illumination $J_{L 1}$ depends on the spectrum of the 


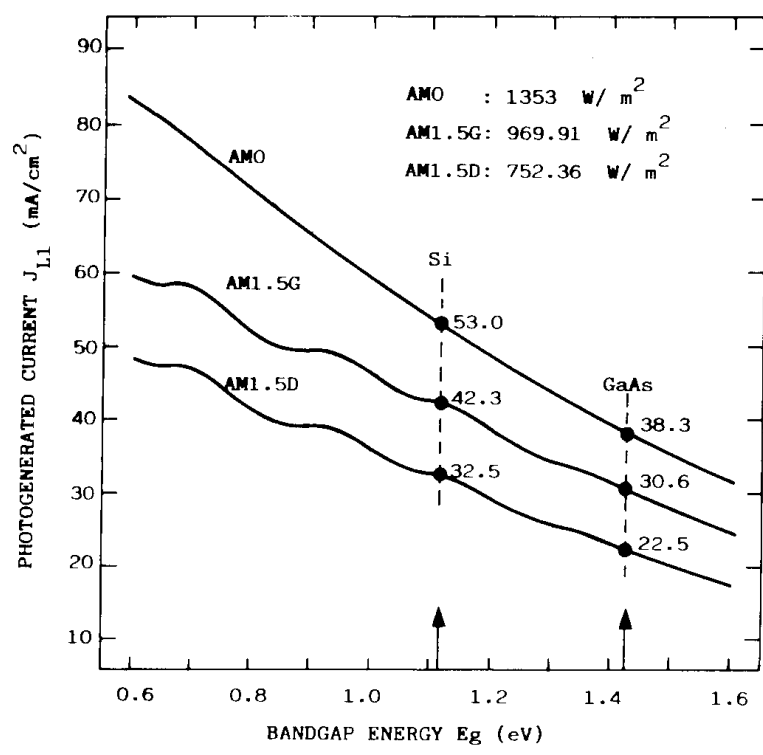

Fig. 1. Maximum photogenerated current as a function of the semiconductor bandgap energy, for different illumination spectra.

incident radiation and is a decreasing function of the semiconductor bandgap $E_{G}$ (Fig. 1). For the calculation of the luminecent losses, an approximate analytical solution of (3) can be found to be

$$
J=J_{L E 0}[\exp (q V / k T)-1]
$$

with

$$
J_{L E 0}=q r(\theta) \frac{2 \pi k T E_{G}^{2}}{h^{3} c^{2}} \exp \left(-E_{G} / k T\right) \equiv r(\theta) A_{0} \exp \left(-E_{G} / k T\right)
$$

or

$$
J_{L . E Y}=\operatorname{qr}(\theta) f\left(E_{G}\right) n_{i}^{2}, \quad f\left(E_{G}\right) \equiv \frac{h^{3} E_{G}^{2}}{c^{2}(4 \pi k T)^{2}\left(m_{c} m_{v}\right)^{3 / 2}}
$$

with $r(\theta)=\sin ^{2} \theta$, for D1-structures and $r(\theta)=\sin ^{2} \theta+n^{2}$ for D2-structures. $m_{c}$ and $m_{v}$ are the electron and hole effective masses, respectively.

The angular selectivity included in our analysis represents a generalization with respect to previously reported works. Thus D1-structures with no angular selectivity, $\theta=\pi / 2$, have been considered in [5] and D2-structures, with the same restrictions, in [3].

The calculation of the open-circuit voltage is straightforward and gives

$$
V_{o c}=\frac{E_{G}}{q}-\frac{k T}{q} \ln \frac{A_{0}}{J_{L 1}} \frac{\sin ^{2} \theta}{X}
$$

for D1-structures. As can be noted, the performance of the cells is obtained as a function of the concentration and the solid angle $2 \theta$. But the concentration ratio and the solid angle are related. From the brightness conservation theorem, it can be demonstrated that the achievable concentration ratios are limited by the following relationship:

$$
X \leq \frac{\sin ^{2} \theta}{\sin ^{2} \theta_{s}} \text { or } K \equiv \frac{X}{\sin ^{2} \theta} \leq \frac{1}{\sin ^{2} \theta_{s}}
$$

if the incident optical medium is air and where $\theta_{s}$ is the solid semiangle of the sun. For $\theta_{s}=0.267^{\circ}$, (9) establishes a maximum value of $X=46050$ for the highest achievable concentration.

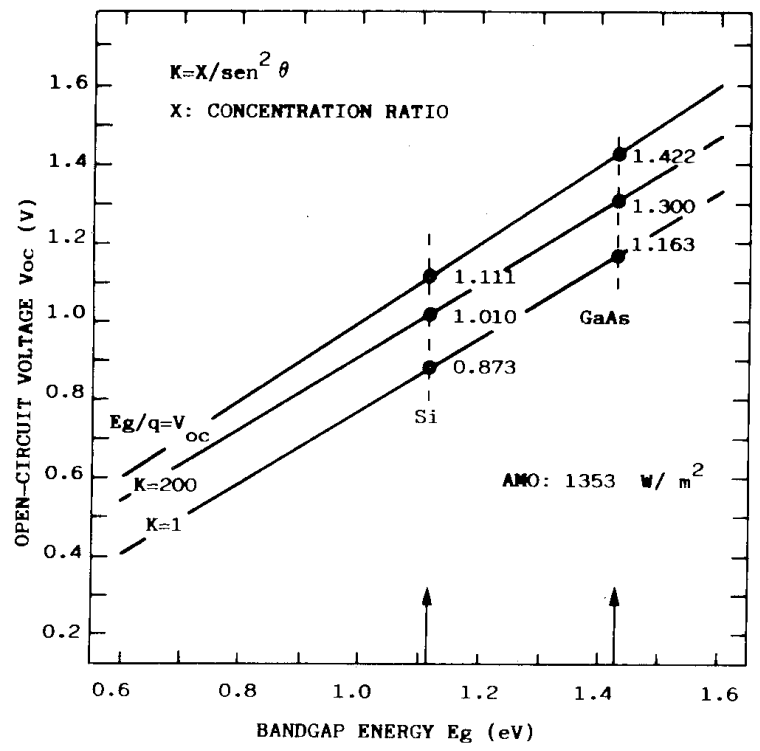

Fig. 2. Open-circuit voltage as a function of the bandgap energy. Corresponds to the maximum photogenerated current given in Fig. 1 and takes into account radiative recombination only. The results are shown for AMO spectrum $(T=300 \mathrm{~K})$.

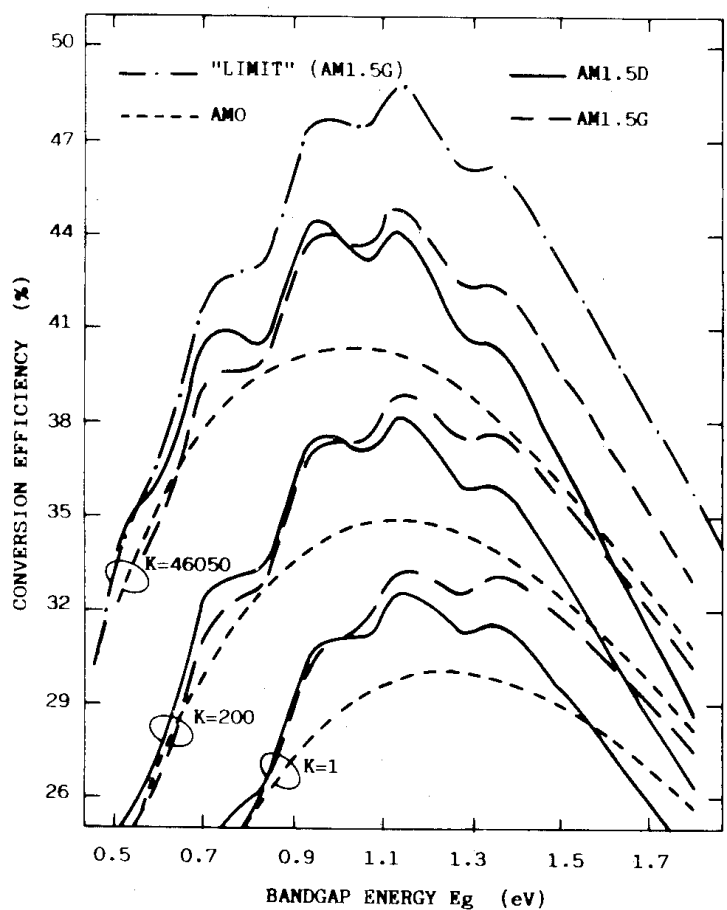

Fig. 3. Conversion efficiencies as a function of the bandgap energy, for the photocurrents of Fig. 1 and radiative recombination only. The curve labeled "limit" corresponds to the case $V_{o c}=E_{G} / k T$ and $F F=1(T=$ $300 \mathrm{~K}$ ).

In the way we have written (8), the dependence of $V_{o c}$ on $X$ and $\theta$ is explicitly shown. It is important to observe that the dependency is produced through the ratio $X / \sin ^{2} \theta$ and therefore the same result can be obtained by increasing $X$ as by decreasing $\theta$ (Fig. 2). 
As the fill factor varies only slightly with $V_{o c}$, the shape of the variation of the solar cell efficiency is almost entirely determined by the product $J_{L} V_{o c}$ and, as $J_{L}$ decreases with $E_{G}$ and $V_{o c}$ increases, the efficiency shows a maximum, as can be seen in Fig. 3. Again, different spectra have been considered and different values of $X / \sin ^{2} \theta$ have been taken as a parameter. The absolute limit for the efficiency has also been calculated by fixing the $V_{o c}$ value to $E_{G}$ and considering a fill factor of unity.

It is interesting to note that the curves of Fig. 3 show a shift of their maximum efficiency to lower values of $E_{G}$ as the parameter $X / \sin ^{2} \theta$ increases. At high values of that parameter, the optimum material lies in the range of 1.1-1.2 eV, where $\mathrm{Si}$ is located. However for $\mathrm{Si}$, and other indirect gap materials, Auger recombination poses a more restrictive limit than the radiative one. So, the advantages predicted in Fig. 3 should be more conveniently approached by direct-gap materials of the same energy gap, for example some ternaries of the InGaAs family.

\section{Auger Recombination versus Luminescent Emission LOSSES}

Since we are more interested in the high-concentration cases, the cell will most likely operate in high injection conditions and, consequently, the ultimate limiting mechanism for a given semiconductor will be determined by the relationship between the luminescent losses and the Auger losses corresponding to high injection. The Auger recombination given by (4) tends to settle towards an asymptote of the form $J=J_{A H 0} \exp (q V / m k T)$. For high injection

$$
J_{A H 0}=q W\left(C_{n}+C_{p}\right) n_{i}^{3} \text { and } m=2 / 3 .
$$

By comparing (7) and (10) it can be seen that Auger and luminescent losses will be equal for a current density

$$
J_{X}=\frac{L_{L E 0}^{3}}{J_{A H 0}^{2}}=q \frac{\left[r(\theta) f\left(E_{G}\right)\right]^{3}}{\left[W\left(C_{n}+C_{p}\right)\right]^{2}} .
$$

For $J$ greater than $J_{X}$, the Auger recombination is dominant and for $J$ lower than $J_{X}$, the radiative recombination dominates. For GaAs, $f\left(E_{G}\right)$ is greater than for $\mathrm{Si}$ while the value of the denominator tends to be lower. Therefore, $J_{X}$ is much higher for GaAs than for Si. By considering $C_{n}=1.22 \times 10^{-30}, C_{p}=0.44 \times 10^{-30}$ $\mathrm{cm}^{6} \cdot \mathrm{s}^{-1}$ [9], and $n_{i}=1.21 \times 10^{10} \mathrm{~cm}^{-3}$, for Si and $C_{n}=1.8 \times$ $10^{-31}, C_{p}=1.2 \times 10^{-30} \mathrm{~cm}^{6} \cdot \mathrm{s}^{-1}[10]$, and $n_{i}=1.67 \times 10^{6}$ $\mathrm{cm}^{-3}$ for GaAs, a value of $J_{X}=5 \times 10^{3} \mathrm{~A} / \mathrm{cm}^{2}$ can be calculated for a GaAs sample $2.5 \mu \mathrm{m}$ thick and a value $J_{X}=5.9 \times 10^{-7}$ $\mathrm{A} / \mathrm{cm}^{2}$ for a Si sample $20 \mu \mathrm{m}$ thick $(r(\theta)=1$ in both cases $)$ Hence it is clear why radiative recombination will constitute the most severe intrinsic limit for GaAs cells while Auger is the limit for Si cells. For very angular selective GaAs cells, however, $r(\theta)$ decreases and for low enough values of $\theta$, the value of $J_{X}$ can decrease so much that the Auger recombination could dominate, even for GaAs cells.

\section{Continuously Varying Absorbance: The Case of GaAs}

For real semiconductors the absorption coefficient $\alpha$ will always be a continuous function of the energy and so will be the absorbance. For a cell with a D1-structure $a(E, \varphi)=1-\exp$ $(-2 \alpha \mathrm{W} / \cos \varphi)$, where the factor 2 accounts for the double pass of the light ray due to the reflecting back surface. For cells with D2-structure $a(E, \varphi)=1-\exp (-\alpha W / \cos \varphi)$. Taking these expressions into account for absorbance, the evaluation of the currents according to (2) and (3) will result in values of $J_{L 1}$ and $J_{l e}$ dependent on $W$ and $\theta$. The calculations can be further simplified by considering a $\varphi$-independent average absorbance $a(E)$ within the solid angle of acceptance.

For the cases for which the SRH and Auger components can be disregarded, the efficiency tends to saturate at high values of thickness. This leads to approximately the same values of efficiency calculated before for $a=1$. The Auger recombination, however, is an unavoidable intrinsic process and consequently there is always

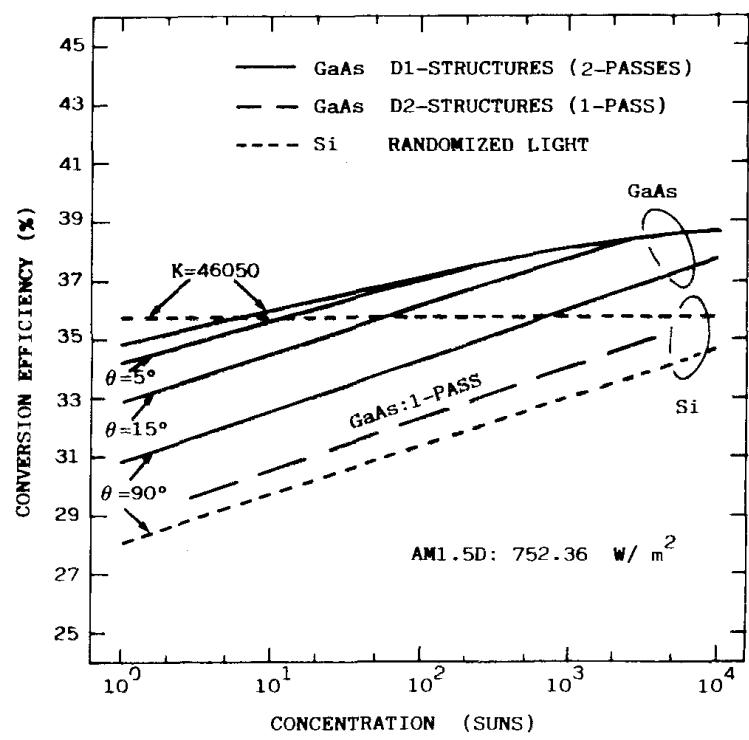

Fig. 4. Conversion efficiencies of $\mathrm{GaAs}$ and $\mathrm{Si}$ solar cells as a function of concentration ratio, with the acceptance angle taken as a parameter. The values were calculated for continuously varying absorbance taking into account radiative and Auger recombinations $(T=300 \mathrm{~K})$.

a finite thickness for the semiconductor slab corresponding to a maximum value of the efficiency smaller than that of unity absorbance. However, as shown in Fig. 4, the performance of a GaAs cell is significantly degraded for Auger recombination only for highly nonisotropical GaAs cells for which the luminescent losses are strongly reduced.

In Fig. 4, the calculated limiting efficiencies of GaAs cells are also compared to those of $S i$ cells, calculated considering an absorbance of $a(E)=\alpha /\left[\alpha+\sin ^{2} \theta / 4 n^{2} W\right]$ as proposed in [6] for fully randomized trapped light. Our results for Si are slightly lower than those reported in [6] because the Auger coefficients taken from [9] are slightly higher. The absorption coefficient for Si was taken from [11] and that for GaAs from the table given in [12].

\section{Recombination Via Defects and Surfaces: Practical CONSIDERATIONS}

To take advantage of this superior potential, however, it is necessary to get rid of the undesirable recombination via defects and surfaces that can dominate in practical GaAs cells. By comparing the luminescent losses, (3), to the asymptotic behavior of the viatrap recombination losses given by (5), it is found that the following conditions must hold, at open-circuit operation, so that the radiative recombination dominates:

$$
\tau_{s r h} N_{B}>\frac{q n_{i}^{2} W}{J_{L E 0}} \quad \text { (for low injection operation) }
$$

and

$$
\tau_{s r h}>\frac{q n_{i} W}{2\left(J_{L E 0} J_{\mathrm{L}}\right)^{1 / 2}} \quad \text { (for high injection) }
$$

It must be emphasized that the lifetimes we are referring to are nonradiative due to recombination via defects exclusively and that the condition just established assures that such a recombination would become negligible as compared with the combination of both radiative recombination and reabsorption processes.

For devices with an absorption region $2.5 \mu \mathrm{m}$ thick, a requirement $\tau_{s r h} N_{B}>1.1 \times 10^{20} \mathrm{~ns} \cdot \mathrm{cm}^{-3}$ results, for the low-injection case. For high-injection operation, the required $\tau$ decreases with the photocurrent and therefore condition (12b) becomes less re- 
strictive as the concentration increases. For a concentration ratio of 1000 suns, the required lifetime is on the order or $200 \mathrm{~ns}$. From the quality of present materials, $\tau N_{B} \approx 5 \times 10^{18} \mathrm{~ns} \cdot \mathrm{cm}^{-3}$ and $\tau\left(\right.$ low $\left.N_{B}\right) \approx 10 \mathrm{~ns}$. It is clear, therefore, that the requirements to approach the intrinsic limits are very demanding. But they are not outside practical possibilities, especially if we deal with the case of high-concentration systems, since values of $\tau N_{B} \approx 10^{19} \mathrm{~ns}$. $\mathrm{cm}^{-3}[13]$ and $\tau\left(\right.$ low $\left.N_{B}\right) \geq 200 \mathrm{~ns}$ have already been experimentally measured [14]

As (12a) and (12b) show, there is still the possibility of reducing the defect-dependent recombination losses by decreasing the thickness of the active region of the device, provided that some lighttrapping procedure is used simultaneously. This approach, however, leads to very thin layers for which some of the effects not considered in our model, such as series resistance, could become very serious. A detailed study of this approach is out of the scope of this study.

At cell surfaces, the asymptotic behavior of (6) for low-injection operation can also be compared to the luminescent losses. These losses will dominate if

$$
S<\frac{J_{L E 0} N_{B}}{q n_{i}^{2}} .
$$

For $N_{B}=5 \times 10^{17} \mathrm{~cm}^{-3}$ the bound would be about $1100 \mathrm{~cm} / \mathrm{s}$. Again this value is not outside practical possibilities because lower surface recombination velocities have been measured experimentally.

\section{Conclusions}

The most important conclusions derived from our work can be summarized as follows:

1) The limiting efficiencies of both GaAs and Si can be raised to roughly $39 \%$ at high concentrations AM1.5D illumination, by using techniques for restricting the angle of acceptance.

2) The potential of GaAs solar cells is clearly higher than that of $\mathrm{Si}$ cells for concentration systems because in those conditions of operation GaAs cells are limited by radiative recombination while Si cells are Auger-limited.

3) For small acceptance angles, the radiative recombination losses can be so considerably reduced that Auger recombination losses can become the limiting factor for GaAs as well as for $\mathrm{Si}$ cells. This effect is responsible for the drop in efficiency shown in Fig. 4 for low concentrations and a small acceptance angle.

4) All the above conclusions require a perfect back-surface mirror, which implies a fairly thin device for the case of GaAs. Such a device is technologically feasible, in principle, by using techniques such as CLEFT [15]. For the most common thick device which radiates part of its luminescent emission into the semiconductor substrate, the benefits of angular selectivity are almost entirely lost (see Fig. 4). Nevertheless, efficiencies are high and the simplicity of conventional GaAs structures could compete favorably in practice with the more sophisticated Si cells which would need angle restrictions and light trapping to give the same efficiencies.

In summary, GaAs solar cells represent a qualified candidate for very high concentration systems with an efficiency potential superior to that of Si. Nevertheless, the potential of GaAs cells is far from being exploited. To take advantage of such a potential, better quality materials (higher lifetimes and diffusion lengths) are required in order to get rid of the influence of the recombination losses via defect levels. Alternatively, devices with thinner active layers can be considered together with the use of light trapping techniques; but this approach seems to be much more demanding from a technological point of view.

\section{REFERENCES}

[1] W. Shockley and H. J. Qieser, "Detailed balance of efficiency of p-n junction solar cells," J. Appl. Phys., vol. 32, pp. 510-519, 1961.

[2] C. D. Mathers, "Upper limit of efficiency of photovoltaic solar cells," J. Appl. Phys., vol. 48, pp. 3181-3182, 1977.

[3] C. H. Henry, "Limiting efficiencies of ideal single and multiple gap terrestrial solar cells," J. Appl. Phys., vol. 51, pp. 4494-4500, 1980.

[4] M. A. Green, "Limits on the open-circuit voltage and efficiency of Silicon solar cells imposed by intrinsic Auger processes," IEEE Trans. Electron Devices, vol. ED-31, pp. 671-678, 1984.

[5] T. Triedje, E. Yablonovitch, G. D. Cody, and B. G. Brooks, 'Limiting efficiency of silicon solar cells,"' IEEE Trans. Electron Devices, vol. ED-31, pp. 711-761, 1984.

[6] P. Campbell and M. A. Green, "The limiting efficiency of silicon solar cells under concentrated sunlight,"' IEEE Trans. Electron Devices, vol. ED-33, pp. 234-239, 1986.

[7] C. M. Chong and M. A. Green, "Limiting efficiency of silicon solar cells under highly concentrated sunlight," IEEE Trans. Electron Devices, vol. ED-34, pp. 2351-2352, 1987.

[8] R. A. Sinton and R. M. Swanson, "Increased photogeneration in thin silicon concentrator solar cells," IEEE Electron Device Lett., vol. EDL-8, pp. 547-549, 1987.

[9] - "Limiting recombination in Si concentrator solar cells," in Proc. 7th Europ. Solar Energy Conf., pp. 917-920, 1987.

[10] M. Takeshima, "Effect of Auger recombination on laser operation in GaAlAs," J. Appl. Phys., vol. 58, pp. 3846-3850, 1985.

[11] K. Rajkanan, R. Singh, and J. Schewchun, "Absorption coefficient of silicon for solar cells calculations," Solid-Statate Electron., vol. 22, pp. 793-795, 1979.

[12] E. D. Palik, in Handbook of Optical Constants of Solids, E. D. Palik, Ed. New York, NY: Academic Press, 1985.

[13] R. J. Nelson, "Measurement of $100 \mu \mathrm{m}$ minority carrier diffusion lengths in $\mathrm{p}-\mathrm{GaAs}$ by a new photoluminescent method," Gallium Arsenide and Related Compounds, vol. 45, pp. 256-262, 1978.

[14] R. J. Nelson and R. G. Sobers, "Minority-carrier lifetime and internal quantum efficiency of surface free GaAs," J. Appl. Phys., vol. 49 , pp. 6103-6108, 1978 .

[15] J. C. C. Fan, C. O. Bozler, and R. W. McClelland, "Thin-film GaAs solar cells," in Proc. 15th IEEE Spec. Photov. Conf., pp. 309-325, 1976.

\section{Physical Modeling of Microwave Transconductance and Capacitances in GaAs MESFET's Operated in Velocity Saturation}

\author{
L. G. HIPWOOD
}

\begin{abstract}
An analytical model applicable at high drain voltage is described which yields simple equations for the key small-signal equivalent circuit elements describing the microwave performance of GaAs MESFET's. The depletion region resulting from the gate potential is represented in an idealized shape. Use is made of Gauss' law to obtain the dimensions of this shape. The response of this to applied signals is postulated to represent that within the MESFET to derive the equivalent circuit values of transconductance, input, and feedback capacitance. The behavior of the gate-depletion extension towards the drain, a function of the gate recess, surface potential, and applied potentials,
\end{abstract}

Manuscript received July 19, 1989; revised November 14, 1989. This work has been carried out with the support of Marconi Defence Systems and the Procurement Executive, Ministry of Defence, sponsored by RSRE. This work forms part of the IEPG TA1 RFP2 project on GaAs MMIC sensitivity analysis. The review of this brief was arranged by Associate Editor S. E. Laux.

The author is with GEC Hirst Research Centre, Marconi Microwave Laboratory, Wembley, Middlesex, HA9 7PP, England.

IEEE Log Number 9034404 